\title{
Avaliação Preliminar do Programa de Humanização no Pré-Natal e Nascimento no Brasil
}

\author{
Preliminary Evaluation of the Prenatal and Birth Humanization Program in Brazil
}

Suzanne Jacob Serruya ${ }^{1}$, Tânia de Giácomo do Lago $^{1}$, José Guilherme Cecatti ${ }^{2}$

\begin{abstract}
RESUM0
Objetivo: avaliar a experiência da implantação do Programa de Humanização no Pré-natal e Nascimento (PHPN) no Brasil, por meio de estudo descritivo, populacional, com informações referentes aos anos de 2001 e 2002.

Métodos: o estudo foi realizado a partir da análise documental e dos dados gerados no SISPRENATAL, avaliando comparativamente entre os estados, regiões e período, os indicadores relativos aos critérios para o acompanhamento pré-natal.

Resultados: até o final de 2002, 3.983 municípios aderiram no país (72\% de adesão) e, destes, $71 \%$ apresentaram produção, constituindo banco de dados com 720.871 mulheres. Em 2002, apenas 28\% das gestantes estavam inscritas, das quais 25\% até 120 dias de gestação. Cerca de $22 \%$ das mulheres tiveram seis consultas, 6\% a consulta puerperal e os exames obrigatórios, apenas 4\% também foram submetidas ao teste para HIV e receberam vacina antitetânica, e 12\% tiveram dois exames para a sífilis. Houve importantes variações regionais, geralmente com melhores indicadores para as regióes Sudeste e Sul.

Conclusões: apesar de os indicadores de qualidade de assistência mostrarem melhora de 2001 para 2002, os baixos percentuais registrados ratificam a necessidade de permanentes avaliações e novas intervenções com o objetivo de melhorar a qualidade desta atenção, prioritariamente nas regiões Norte e Nordeste.
\end{abstract}

PALAVRAS-CHAVE: Pré-natal. Vacinação. Atendimento primário. Puerpério. Indicadores em saúde.

\section{Introdução}

A medicalização e instrumentalização do parto, que constituíram a característica marcante da atenção obstétrica do século XX no mundo ocidental, fazem parte da institucionalização do poder médico que, no corpo feminino, atuou fundamentalmente normatizando a reprodução ${ }^{1}$. Des-

\footnotetext{
'Área Técnica da Saúde da Mulher, Ministério da Saúde, Brasília, DF (até Janeiro de 2003)

${ }^{2}$ Departamento de Tocoginecologia, Faculdade de Ciências Médicas, Universidade Estadual de Campinas (UNICAMP) Correspondência:

José Guilherme Cecatti

Caixa Postal 6030

13081-970 - Campinas - SP

e-mail: cecatti@unicamp.br
}

te modo, mais recentemente, a mudança do local de ocorrência do parto e a participação, cada vez mais atuante, dos profissionais de saúde caracterizam de forma marcante a experiência do parto. Provavelmente a razão principal para a ocorrência destas mudanças foi a alta mortalidade materna e perinatal ${ }^{1,2}$. Sem dúvida, um dos principais benefícios dela resultante foi a melhora destes resultados. À medida que determinadas técnicas e procedimentos foram sendo incorporados, a mortalidade de mulheres e recém-nascidos caiu significativamente e hoje, em países desenvolvidos, a morte materna é rara e incomum $^{3-6}$.

A atenção que se concentrava no parto estendeu-se ao período anterior ao nascimento, ini- 
ciando-se no fim do século XIX a chamada assistência materno-infantil, representada por consultas no período pré-natal esparsas, sem sistematização clínica. Em quase todos os países a prática de pré-natal foi adotada e, no Brasil, era o tipo de assistência mais oferecido às mulheres até o final da década de 70. A partir deste momento os grupos de mulheres e os de saúde reivindicavam a ampliação da assistência à mulher a partir de movimento articulado com a proposta de reforma sanitária e a criação de sistema único de saúde, público e universal ${ }^{7}$. O movimento sanitário, tomando a saúde como um direito inalienável, exigia que o Estado reordenasse o sistema de saúde com base nos princípios de universalidade, eqüidade e integralidade.

Foi neste contexto que o Ministério da Saúde lançou as bases programáticas do PAISM, Programa de Assistência Integral à Saúde da $\mathrm{Mu}$ 1 her ${ }^{8}$, em 1984. O PAISM deveria prover ações relacionadas à gravidez, contracepção, esterilidade, prevenção de câncer ginecológico, diagnóstico e tratamento das doenças sexualmente transmissiveis, sexualidade, adolescência e climatério $^{7}$. Apesar de ter representado avanço significativo em termos de direitos reprodutivos para as mulheres brasileiras, sua implementação sofreu dificuldades políticas, financeiras e operacionais, o que impediu na prática que o Programa se transformasse, de fato, em agente transformador da saúde de todas as mulheres. Para se ater apenas à atenção pré-natal, alguns dados disponíveis mostram que os indicadores de qualidade em saúde durante o pré-natal no final da década de noventa deixavam muito a desejar para um país com os recursos financeiros e humanos e com um programa de saúde como o Bra$\mathrm{sil}^{9}$.

Assim, reconhecendo a necessidade de estabelecer nova estratégia, em junho de 2000, o Ministério da Saúde instituiu o Programa de Humanização no Pré-Natal e Nascimento (PHPN), que apresenta em sua formulação os seguintes objetivos: reduzir as altas taxas de morbimortalidade materna e perinatal, ampliar o acesso ao prénatal, estabelecer critérios para qualificar as consultas e promover o vínculo entre a assistência ambulatorial e o parto $^{10}$.

O estabelecimento de critérios buscava prover os municípios de um modelo básico para a assistência, com as atividades consideradas indispensáveis. O enfoque era que cada mulher realizasse todas as ações e não que a assistência fosse pensada como a soma delas, ou seja, a idéia de qualidade da atenção humanizada era centrada individualmente em cada mulher que deveria receber todos os critérios mínimos previamente determinados, e não no coletivo por meio do total de consultas ou do total de cada exame realizado no âmbito do Programa. A mudança desta lógica é fundamental, uma vez que até então a questão de produção de serviços, consultas e exames não considerava o impacto que a assistência devia ter. Como os dados nos sistemas de informação seguem uma lógica quantitativa, estão comprometidas sistematicamente as avaliações qualitativas. A compreensão de que somente o conjunto das ações reflete integralidade e pode produzir algum impacto, e que seria então necessário garantir a realização destas atividades com qualidade, fundamentava esta estratégia.

Deste modo, foram estabelecidos para o cuidado pré-natal vários critérios relativos à captação precoce da gestante e à realização de um mínimo de procedimentos considerados essenciais e que pudessem ser implementados na grande maioria dos municípios brasileiros (v. Tabela 1). Apesar de o PHPN ter sido instituído há mais de três anos, ainda não havia sido realizada uma análise mais ampla, quantitativa e qualitativa, da atenção pré-natal no país como um todo, mas apenas em alguns municípios isolados, demonstrando ainda déficits importantes na implementação dos critérios mínimos exigidos pelo Programa $^{11,12}$. Isso permitiria também avaliação inicial desta intervenção e de seu respectivo sistema de informação. Assim, o objetivo deste estudo foi avaliar a experiência da implantação do PHPN no Brasil, seu processo de implantação, o sistema criado para o gerenciamento de dados gerados (SISPRENATAL) e os indicadores preliminares do sistema para as distintas regiões e estados, comparativamente nos dois anos iniciais de seu funcionamento.

\section{Material e Método}

A proposta do presente estudo foi de abordar epidemiologicamente a implantação do PHPN durante o período de dois anos a partir de sua instituição (2001 e 2002), contemplando a totalidade de municípios que aderiram ao Programa (3983) e de mulheres nele registradas (720.871). Portanto, por se tratar de uma avaliação do Programa em todos os estados, a abordagem é populacional. Foram incluídos os dados gerados a partir do sistema de informações, criado especificamente para o gerenciamento do Programa (SISPRENATAL), de todos os municípios e ges- 
tantes cadastradas no sistema que tem como fonte de dados dois documentos: Ficha de Cadastramento da Gestante e a Ficha de Registro Diário dos Atendimentos da Gestante. Estas informações originadas nas unidades básicas de saúde são incluídas no sistema mensalmente, e então o SISPRENATAL gera o Boletim de Produção Ambulatorial - BPA, para importação no Sistema de Informações Ambulatoriais do SUS SIA/SUS. Participaram da presente avaliação apenas os municípios que aderiram ao Programa e que também informaram sua produção no sistema.

Para acompanhar as gestantes foi elaborado um sistema informatizado, o SISPRENATAL, de uso obrigatório para os municípios. Para pagamento do incentivo relativo ao cadastramento ( $\mathrm{R} \$ 10,00$ reais por gestante cadastrada), a primeira consulta deveria ser realizada com até 120 dias de idade gestacional (captação precoce). Para pagamento do incentivo relativo à conclusão ( $\mathrm{R} \$ 40,00$ reais por gestante), o município deveria cumprir todos os critérios estabelecidos: seis ou mais consultas de pré-natal, todos os exames obrigatórios, a imunização antitetânica e a consulta puerperal. A criação e o desenvolvimento de um sistema de informações sobre a assistência pré-natal tinham como objetivo primeiro e circunstancial monitorar o cumprimento dos critérios estabelecidos no PHPN. O objetivo central, no entanto, era disponibilizar, definitivamente, instrumento que permitisse ao gestor acompanhar a assistência prestada nos diferentes níveis de gestão e obter indicadores de processo e resultados sobre a atenção pré-natal, induzindo as intervenções no processo de atenção. Para este acompanhamento da assistência, o sistema fornece um conjunto de relatórios e indicadores de resultados e de processo.

O primeiro indicador gerado pelo sistema foi o de cobertura, referente ao percentual de gestantes inscritas no Programa e que tiveram a primeira consulta, em relação ao número de nascidos vivos no período.

As fontes para a coleta de informações foram relatórios e planilhas do Programa, portarias relativas ao Programa, material instrucional para sua divulgação, planilha de controle de adesão, Ficha de Cadastramento da Gestante, Ficha de Registro Diário de Atendimento, planilhas de controle de produção, relatórios emitidos no SISPRENATAL, dados do Sistema de Informação Ambulatorial (SIA-DATASUS) dados do Sistema de Informação Hospitalar (SIH-DATASUS), e finalmente dados do Sistema de Informação de Nas- cidos Vivos (SINASC). Os resultados são apresentados na forma de porcentuais de gestantes cadastradas que atenderam os critérios mínimos para cada período, comparados entre os diferentes estados e regiões do Brasil. O projeto para o presente estudo foi avaliado e aprovado pelo Comitê de Ética em Pesquisa da Faculdade de Ciências Médicas da Universidade Estadual de Campinas.

\section{Resultados}

No primeiro semestre do Programa, o segundo do ano 2000, apenas quatro estados encaminharam termos de adesão, totalizando $55 \mathrm{mu}-$ nicípios. De janeiro a junho de 2001, 586 municípios aderiram ao Programa e no final do segundo semestre 2.523 municípios já o haviam feito. Neste momento, apenas uma capital não havia aderido, e as regiões Norte e Centro-Oeste tinham os menores percentuais $(4,4$ e $14,6 \%$, respectivamente). A adesão continuou agregando municípios e no final de 2002, em alguns estados, a adesão atingia totalidade dos municípios e em onze estados o percentual de adesão era superior a $80 \%$. Os estados com baixo percentual, inferior a 25\%, eram Espírito Santo, Maranhão e Rio Grande do Sul. Em dezembro de 2002, 3983 municípios, representando $71 \%$ do total do Brasil, já haviam aderido.

Em 2001, dois estados não apresentaram nenhum cadastro de gestantes: Amazonas e Espírito Santo. Os estados que alcançaram maior cobertura foram: Paraná, Sergipe e Paraíba, superior a 20\% (Tabela 1). Em 2002, todos os estados produziram dados e houve aumento considerável no número de mulheres cadastradas em vários estados, apesar de a cobertura estar muito aquém do esperado, quase sempre inferior a $50 \%$. Os estados com maior cobertura foram Paraná, Paraíba, Tocantins, Minas Gerais e Santa Catarina, superiores a $30 \%$. Os estados com menor cobertura foram Acre, Rio de Janeiro e Pernambuco, em sua maioria inferiores a $10 \%$. No primeiro ano do Programa, praticamente a totalidade de mulheres cadastradas tinha até 120 dias de gestação (captação precoce), sendo o resultado do percentual de cobertura igual ao da captação precoce. Em 2002, apesar da permissão de cadastramento da mulher em qualquer idade gestacional, o percentual encontrado, de $24,5 \%$, foi muito próximo do percentual de cobertura, 27,9\% (Tabela 1). 
Tabela 1 - Critérios para a atenção pré-natal estabelecidos pelo Programa de Humanização no Pré-Natal e Nascimento (PHPN).

1. Primeira consulta até $04^{\circ}$ mês de gestação.

2. Garantir a realização dos seguintes procedimentos:

2.1. No mínimo, seis consultas de pré-natal, sendo, preferencialmente, uma no primeiro trimestre, duas no segundo trimestre e três no terceiro trimestre da gestação.

2.2. Uma consulta no puerpério, até quarenta e dois dias após o nascimento.

2.3. Exames laboratoriais:

a) $A B O-R h$, na primeira consulta;

b) VDRL, um exame na primeira consulta e outro próximo à trigésima semana da gestação;

c) Urina rotina, um exame na primeira consulta e outro próximo à trigésima semana da gestação;

d) Glicemia de jejum, um exame na primeira consulta e outro próximo à trigésima semana da gestação;

e) Hemoglobina/hematócrito, na primeira consulta.

2.4. Oferta de teste de HIV, com um exame na primeira consulta, naqueles municípios com população maior que cinqüenta mil habitantes.

2.5. Aplicação de vacina antitetânica até a dose imunizante (segunda) do esquema recomendado, ou dose de reforço em muIheres já imunizadas.

2.6. Atividades educativas.

2.7. Classificação de risco gestacional a ser realizada na primeira consulta e nas consultas subseqüentes.

2.8. Atendimento às gestantes classificadas como de risco, garantindo o vínculo e acesso à unidade de referência para atendimento ambulatorial e/ou hospitalar à gestação de alto risco.

Brasil, 200010

Cerca de 20\% das mulheres cadastradas nos dois anos do Programa tiveram seis ou mais consultas no pré-natal. Em 2001, apenas seis estados apresentaram dados neste indicador: São Paulo, Paraná, Sergipe, Distrito Federal, Goiás e Ceará. Em 2002, os percentuais superiores ao percentual nacional foram relatados no Paraná, São Paulo e Paraíba. Neste ano, não apresentaram resultados: Amazonas, Espírito Santo e Rio Grande do Sul (Tabela 2). A Tabela 3 apresenta também o percentual de gestantes que atingiu os seguintes critérios: seis ou mais consultas de pré-natal, os exames básicos e a consulta de puerpério. Este percentual apresentou-se ainda mais reduzido, apenas 2,8\% das mulheres em 2001 em somente três estados: São Paulo, Paraná e Sergipe. Em 2002, três esta- dos apresentaram percentuais superiores ao percentual nacional, 5,6\%: Paraná, Paraíba e Rondônia. Em cinco estados o percentual foi igual a zero, ou seja, nenhuma gestante cadastrada atingiu o somatório destes critérios: Amazonas, Amapá, Roraima, Espírito Santo e Rio Grande do Sul.

Tabela 2 - Percentual de gestantes inscritas que tiveram (A) seis ou mais consultas de pré-natal ou (B) seis ou mais consultas de pré-natal, a de puerpério e todos os exames básicos, em 2002, por Unidade da Federação.

\begin{tabular}{|c|c|c|}
\hline Estados & A: $\geq 6$ consultas & $\begin{array}{c}\mathrm{B}: \geq 6 \text { consultas+ } \\
\text { puérperas }+ \text { exames }\end{array}$ \\
\hline \multicolumn{3}{|l|}{ Região Norte } \\
\hline Acre & 9,9 & 4,6 \\
\hline Amapá & 5,3 & - \\
\hline Amazonas & - & - \\
\hline Pará & 10,2 & 2,5 \\
\hline Rondônia & 21,9 & 5,9 \\
\hline Roraima & 13,3 & - \\
\hline Tocantins & 13,2 & 3,1 \\
\hline \multicolumn{3}{|l|}{ Região Nordeste } \\
\hline Alagoas & 16,5 & 4,4 \\
\hline Bahia & 2,7 & 0,1 \\
\hline Ceará & 22,5 & 5,3 \\
\hline Maranhão & 8,3 & 2,9 \\
\hline Paraíba & 22,6 & 6,1 \\
\hline Pernambuco & 3,4 & 0,3 \\
\hline Piauí & 21,7 & 4,7 \\
\hline Rio Grande do Norte & 9,9 & 2,0 \\
\hline Sergipe & 12,7 & 2,3 \\
\hline \multicolumn{3}{|l|}{ Região Centro-Oeste } \\
\hline Distrito Federal & 5,9 & 0,7 \\
\hline Goiás & 11,2 & 3,7 \\
\hline Mato Grosso & 12,3 & 2,2 \\
\hline Mato Grosso do Sul & 3,2 & 0,1 \\
\hline \multicolumn{3}{|l|}{ Região Sudeste } \\
\hline Espírito Santo & - & - \\
\hline Minas Gerais & 13,2 & 2,6 \\
\hline Rio de Janeiro & 19,1 & 3,5 \\
\hline São Paulo & 28,0 & 5,4 \\
\hline \multicolumn{3}{|l|}{ Região Sul } \\
\hline Paraná & 38,3 & 8,8 \\
\hline Santa Catarina & 5,2 & 0,2 \\
\hline Rio Grande do Sul & - & - \\
\hline Brasil & 22,6 & 5,6 \\
\hline
\end{tabular}


Tabela 3 - Percentual de gestantes inscritas no PHPN que tiveram a primeira consulta em qualquer período ou até 120 dias de gestação, em relação ao número de nascidos vivos no período, por Unidade da Federação.

\begin{tabular}{|c|c|c|c|c|}
\hline \multirow[t]{2}{*}{ Estados } & \multicolumn{2}{|c|}{2001} & \multicolumn{2}{|c|}{2002} \\
\hline & $1^{\text {a }}$ consulta & $\begin{array}{l}1^{\text {acconsulta }} \\
\text { até } 120 \text { dias }\end{array}$ & $1^{\text {a }}$ consulta & $\begin{array}{l}1^{\text {acconsulta }} \\
\text { até } 120 \text { dias }\end{array}$ \\
\hline
\end{tabular}

Região Norte

Acre

Amapá

Amazonas

Pará

Rondônia

Roraima

Tocantins

Região Nordeste

Alagoas

Bahia

Ceará

Maranhão

Paraíba

Pernambuco

Piauí

Rio Grande do Norte

Sergipe

Região Centro-Oeste

Distrito Federal

Goiás

Mato Grosso

Mato Grosso do Sul

Região Sudeste

Espírito Santo

Minas Gerais

Rio de Janeiro

São Paulo

Região Sul

Paraná

Santa Catarina

Rio Grande do Sul

Brasil

Ao indicador apresentado na coluna A da Tabela 4, está agregada a realização do exame HIV, com todos os outros critérios. Como a realização deste exame necessita de consentimento informado, este percentual foi bastante reduzido para todas as regiões do Brasil. Na coluna B desta mesma tabela são apresentados os percentuais relativos ao teste do HIV que mostram que as regiões Sul e Sudeste apresentaram a maior média naci-

onal. Em 2002 todos os estados apresentaram resultados, com destaque para quatro deles: Santa Catarina, Paraná, São Paulo e Rondônia. Considerando a importância do exame sorológico para o diagnóstico e prevenção da sífilis congênita, a realização de dois exames de VDRL é considerada separadamente neste indicador. Em 2002, apesar da participação de todos os estados, o percentual caiu para $12,1 \%$, destacando-se os estados de Rondônia, Rio de Janeiro e Santa Catarina, com os maiores percentuais, ao redor de $10 \%$.

Tabela 4 - Percentual de gestantes inscritas no Programa. A - Completo: tiveram seis ou mais consultas de pré-natal, a de puerpério, todos os exames básicos, o teste HIV, a $2^{\text {a }}$ dose ou a de reforço ou a imunizante da vacina antitetânica; B - submetidas ao teste HIV; e C - 2 testes para VDRL, por Unidade da Federação, em 2002.

\begin{tabular}{llll}
\hline Estados & A & B & C
\end{tabular}

Região Norte

Acre

Amapá

Amazonas

Pará

Rondônia

Roraima

0,4

13,1

4,4

Tocantins

-

-

1,5

8,3

5,2

Região Nordeste

Alagoas

Bahia

Ceará

Maranhão

Paraíba

Pernambuco

5,5

$$
4,7
$$

Piauí

Rio Grande do Norte

Sergipe

$$
6,2
$$

4,9

Região Centro-Oeste

Distrito Federal

Goiás

Mato Grosso

Mato Grosso do Sul

$$
1,7
$$

37,7

11,0

Região Sudeste

Espírito Santo

Minas Gerais

Rio de Janeiro

São Paulo

Região Sul

Paraná

Santa Catarina

Rio Grande do Sul

Brasil

1,

1,6

20,7

4,7

5,3

, 7

15,3

6,9

0,0

11,5

6,4

1,2

12,4

6,7

0,5

10,0

5,1

1,0

0,1

20,8

7,1

1,9

0,7

9,6

7,4

1,0

12,3

6,3

0,4

14,9

7,4

9,3

4,5

0,7

18,6

5,2

1,2

21,5

5,9

2,0

28,7

8,3

- $\quad 32,3$

3,2

$\quad 15,0$

7,7

3,1

29,9

9,6

4,4

30,2

7,0

. 


\section{Discussão}

O presente estudo apresenta os resultados preliminares da avaliação nacional do PHPN, apontando as diferenças regionais e estaduais encontradas. Uma primeira avaliação global para o país referente a este mesmo período é motivo de outra publicação, que também mostra o processo de adesão e produção de dados no sistema informativo do Programa ${ }^{13}$.

A maioria dos programas atuais de atenção pré-natal é originária de modelos desenvolvidos em países ocidentais nas primeiras décadas do século passado. Embora parcela significativa tenha calendário e conteúdo similar, seu desenvolvimento apresenta muitas diferenças: em relação aos profissionais envolvidos, às práticas recomendadas e às práticas efetivadas, além das diferentes taxas de adesão de mulheres ${ }^{14,15}$.

Apesar de o pré-natal ser considerado um dos quatro pilares da maternidade segura, poucas avaliações sistemáticas haviam sido realizadas sobre a efetividade destes programas, a fim de determinar quais intervenções teriam melhores resultados $^{14-18}$. Mais recentemente, vários autores têm discutido a importância da assistência pré-natal, seus critérios, as bases científicas das intervenções que são realizadas e as repercussões sobre a saúde materna e perinatal ${ }^{19}$. No Brasil, diferentes avaliações realizadas sobre a atenção pré-natal também têm apontado a necessidade de mudanças, em especial buscando a equidade e a melhoria na qualidade dos serviços, com objetivo de otimizar resultados ${ }^{11,12,20,21}$.

Deste modo, considerou-se fundamental no PHPN estabelecer critérios para determinar quais deveriam ser as práticas assistenciais mínimas, ou seja, aquelas cuja ausência comprometeria a qualidade da assistência. Os critérios foram determinados a partir de dois pressupostos: o de que comprovadamente eram efetivos e o de que operacionalmente eram, em curto prazo, exeqüiveis. Os critérios escolhidos para qualificar a assistência buscavam principalmente fortalecer a prática de valorizar a "boa" e soberana clínica, com procedimentos simples do ponto de vista técnico, mas fundamentais em termos de resul$\operatorname{tados}^{10,22}$.

Assim, o Programa recomendava seis consultas como o número mínimo para cada mulher. Até o momento desta definição no PHPN, não havia estudos conclusivos sobre qual deve ser o número mínimo ou ideal capaz de garantir os resultados esperados ${ }^{16,23}$. A maioria dos estudos consultados fazia referência a pelo menos uma con- sulta no primeiro trimestre, variando a partir daí o número de atendimentos recomendados. Como o país já apresentava uma média de quatro consultas por mulher que tinha seu parto no SUS, e com a suposição de que o incremento de $50 \%$, representado por mais duas consultas, seria possível, este número foi considerado como o que deveria ser recomendado, embora com o reconhecimento de que, regionalmente, este incremento significaria meta difícil a médio e longo prazo, nas regiões Norte e Nordeste.

No estudo multicêntrico da OMS, no qual se avaliou forma alternativa e simplificada de prénatal, o número de consultas recomendado foi de quatro consultas para cada mulher ${ }^{18}$. A opinião das mulheres foi pesquisada por meio de método quantitativo e qualitativo, neste caso utilizando técnicas de coleta de informações através de grupos focais e entrevistas em profundidade ${ }^{24}$. Na análise dos resultados do estudo, as mulheres revelaram-se satisfeitas, em geral, mas mostraram-se preocupadas com o número e o espaçamento das consultas, com o relacionamento dispensado pelos profissionais e a necessidade de obter mais informações sobre alguns temas como nutrição e auto-cuidado ${ }^{23}$.

Avaliando o conjunto dos indicadores do Programa, no período em questão, é possível perceber que houve aumento em todos indicadores. A cobertura do Programa cresceu significativamente, com $70 \%$ de mulheres cadastradas em 2002. Entretanto, leitura vertical dos indicadores, nos dois anos em estudo, aponta que à medida que são agregados os critérios assistenciais, os percentuais diminuem, indicando que é a realização do conjunto das atividades o maior desafio na atenção prénatal. A avaliação do desempenho dos estados e regiões em relação aos indicadores confirmou a idéia de que, onde a organização e gestão dos serviços estão mais bem estabelecidas, coincidentemente com o desenvolvimento econômico e social, geralmente este processo foi menos complicado, mostrando melhores resultados. Isso está de acordo com outras avaliações previamente realizadas do programa a nível municipal ${ }^{11,12}$. Embora as dificuldades no campo político tenham sido relevantes para a implementação do Programa, a falta de amadurecimento de mecanismos institucionais, como a regionalização e a descentralização, somadas à escassez e concentração de recursos, foram os desafios mais difíceis de contornar.

Dados não apresentados mostram redução de cerca de $30 \%$ no número da primeira para a segunda consulta de pré-natal no Programa, sugerindo que provavelmente muitas mulheres, 
neste momento, não têm seu seguimento registrado. Os percentuais seguintes, para três, quatro e cinco consultas, apresentam menores declínios, mostrando que, uma vez sistematizado, a perda de registro é menor. Entretanto, seguramente o número de mulheres que teve seis consultas ou mais no pré-natal no SUS é maior que $20 \%$, uma vez que somente $28 \%$ de todas as mulheres foram cadastradas no SISPRENATAL em 2002.

Quando o Programa foi elaborado, havia muitos indícios de que a realização dos exames básicos que seriam recomendados entre os critérios estabelecidos seria forte ponto de estrangulamento da atenção. Com os registros existentes antes do SISPRENATAL, não era possível saber quantos e quais exames haviam sido realizados especificamente para a atenção pré-natal. Apenas eram conhecidos os totais de exames por categoria (bioquímica, por exemplo), mas não se conseguia relacioná-los a nenhuma assistência em particular. A hipótese de que as mulheres não conseguiam ter acesso aos exames por dificuldade de recursos locais era reforçada por indicadores como o da sífilis congênita e por verificação, in loco, nos municípios. A partir desta constatação é que se sedimentou a idéia de criar um incentivo para que o município fizesse frente às despesas relativas aos exames e, com base em uma previsão de gastos, foi estabelecido um incentivo de $\mathrm{R} \$ 50,00$ por mulher, uma vez que este valor seria suficiente para pagar todos os exames, inclusive a sorologia para HIV, o mais caro entre eles.

Os exames definidos para compor este pacote mínimo foram $\mathrm{Hb} / \mathrm{Ht}$, glicemia de jejum, VDRL, $\mathrm{ABO}-\mathrm{RH}$, exame de urina simples (ou de rotina, ou tipo I) e o HIV. Na maioria dos modelos de assistência pré-natal pesquisados são estes os exames realizados, aparecendo com menos freqüência a glicemia de jejum e o HIV. O exame de glicemia, o VDRL e o exame de urina deveriam ser repetidos por volta da $30^{\underline{a}}$ semana. As razões técnicas e operacionais para a escolha destes exames consideraram a sua importância para a garantia da boa assistência e são consensuais na maioria dos estudos e guias clínicos.

A presença da glicemia de jejum, menos comum nos guias clínicos de conduta, foi estabelecida como norma pelo Ministério da Saúde por recomendação do Consenso Nacional sobre Diabetes e Gravidez ${ }^{22}$, que levou em conta, além das questões epidemiológicas, o fato de que este exame já faz parte da rotina de assistência prénatal. O exame para detecção do HIV, também menos comum nos guias clínicos, obedeceu à mesma lógica. Existiam recomendações da Comis- são Nacional de DST \& AIDS, por razões epidemiológicas, e muitos municípios já ofereciam o exame.

Assim, com o Programa, no contexto da saúde da mulher, foi reconhecida a necessidade da efetivação de uma política que estabelecesse critérios e indicadores capazes de monitorar processos e resultados, a fim de melhorar os resultados maternos e perinatais. A instituição do Programa de Humanização no Pré-natal e Nascimento definiu estratégia de melhoria da qualidade da atenção obstétrica, considerada inadequada, na qual o paradigma conceitual foi a humanização, na perspectiva dos direitos da mulher. Sua implementação proporcionou amplas discussões em muitos municípios e estados, uma vez que se mostrou um processo complexo e indutor de avaliações sobre a assistência pré-natal, em todos seus aspectos: conceituais, formais e de organização da assistência. A instituição do PHPN colocou em discussão a atenção pré-natal, buscando redefinir as bases conceituais e as práticas assistenciais. Este debate esteve em consonância com a discussão de modelos de assistência pré-natal, que tem sido ampla e recorrente, uma vez que esta atenção é praticada em todo mundo.

A elaboração e instalação do sistema de informações sobre o pré-natal, o SISPRENATAL, disponibilizou relatórios e indicadores que permitem um controle, regulação e monitoramento da assistência pré-natal oferecida. Permitiu identificar um retrato da assistência preocupante e desafiador. Apesar das limitações desta estratégia, é importante reconhecer que a possibilidade de ampliar o leque de informações sobre uma atenção tão fundamental possibilitará que cada gestor, no seu nivel de competência, possa tomar as medidas, mais prontamente, na direção de garantir uma assistência com dignidade, pautada por intervenções efetivas, a cada mulher. Isso pode ser exemplificado pela disponibilidade da variação dos indicadores entre as diferentes regiões e estados do país, permitindo que em cada nivel sejam tomadas medidas facilitadoras ao cumprimento dos requisitos do Programa, pressupondo-se que isto redunde em melhoria da qualidade da atenção prénatal às mulheres e, indiretamente, da saúde materna e perinatal do país.

A avaliação preliminar dos indicadores gerados nestes dois anos mostrou que apenas para pequena parcela das mulheres cadastradas houve registro do cumprimento integral dos critérios. A maioria das mulheres recebeu assistência desarticulada e parcial. Menos de $25 \%$ das mulheres cadastradas teve seis ou mais consultas. Os pontos de estrangulamento detectados na 
atenção, como a realização de exames laboratoriais e a consulta puerperal, sinalizam para a necessidade de rever os mecanismos atuais e propor novas medidas, além das necessárias mudanças baseadas em novas evidências. Os percentuais da realização de VDRL e da vacinação antitetânica apontam para a necessidade de reavaliar questões consideradas da "rotina" assistencial, uma vez que o consenso técnico acerca de sua importância não foi traduzido por sua sistemática realização.

\section{ABSTRACT}

Purpose: to evaluate the experience of implementation of the Brazilian Prenatal and Birth Humanization Program (PHPN) in 2001 and 2002, through a population descriptive study.

Methods: the study was performed through documental analysis and using data generated by SISPRENATAL, comparatively evaluating the indicators concerning criteria for prenatal follow-up in different states, regions and period.

Results: until the end of 2002, 3983 municipalities joined the Program (72\% adhesion) and, among them, $71 \%$ reported results, constituting a data base of 720,871 women. In 2002 only $28 \%$ of the pregnant women were already registered, $25 \%$ before 120 days of pregnancy. Nearly 22\% of the women had six prenatal visits, $6 \%$ had the post-partum visit and the compulsory tests performed, only $4 \%$ had also the HIV test and were vaccinated against tetanus, and $12 \%$ had two examinations performed for syphilis. There were important regional variations, generally showing better indicators for the Southeast and South regions.

Conclusions: although the indicators of quality of care showed an improvement from 2001 to 2002, the recorded low percentages attest the need for permanent evaluations and new interventions with the aim of improving the quality of this care, especially in the North and Northeast regions.

KEYWORDS: Prenatal care. Humanization. Perinatal health. AIDS.

\section{Referências}

1. Foucault M. O nascimento da clínica. $5^{\text {a }}$ ed. Rio de Janeiro: Forense Universitária; 1998.
2. Rosenfield A, Maine D. Maternal mortality. A neglected tragedy. Where is the $\mathrm{M}$ in $\mathrm{MCH}$ ? Lancet 1985; 2:83-5.

3. World Health Organization. Care in normal birth. A practical guide. Maternal and Newborn Health/ Safe Motherhood Unit. Geneva: World Health Organization; 1996.

4. WHO/UNFPA/UNICEF/World Bank. Reduction of maternal mortality: a joint statement. Geneva: World Health Organization; 1999.

5. Shiffman J. Can poor countries surmount high maternal mortality? Stud Fam Plann 2000; 3:274-89.

6. Alleyne GA, Castillo-Salgado C, Schneider MC, Loyola E, Vidaurre M. Overview of social inequalities in health in the region of the Americas, using various methodological approaches. Rev Panam Salud Publica 2002; 12:388-97.

7. Costa AM. Desenvolvimento e implantação do PAISM no Brasil. In: Giffin K, Costa SH, editores. Questões da Saúde Reprodutiva. Rio de Janeiro: FIOCRUZ; 1999. p. 319-36.

8. Ministério da Saúde. PAISM. Programa de assistência integral à saúde da mulher. Brasília: Centro de Documentação do Ministério da Saúde; 1984.

9. Serruya S, Lago TG, Cecatti JG. O panorama da atenção pré-natal no Brasil e o Programa de Humanização do Pré-natal e Nascimento. Rev Bras Saúde Materno Infantil. No prelo 2004.

10.Ministério da Saúde. Programa de humanização no pré-natal e nascimento. Brasília: Secretaria de Políticas de Saúde; 2000.

11.Trevisan MR, De Lorenzi DRS, Araujo NM, Ésber K. Perfil da assistência pré-natal entre usuárias do Sistema Único de Saúde em Caxias do Sul. Rev Bras Ginecol Obstet 2002; 24:293-9.

12. Coutinho T, Teixeira MTB, Dain S, Sayd JD, Coutinho LM. Adequação do processo de assistência pré-natal entre as usuárias do Sistema Único de Saúde em Juiz de Fora-MG. Rev Bras Ginecol Obstet 2003; 25:717-24.

13. Serruya S, Cecatti JG, Lago TG. O Programa de Humanização no Pré-Natal e Nascimento (PHPN) do Ministério da Saúde no Brasil: resultados iniciais. Cad Saúde Pública. No prelo 2004.

14.Lumbiganon P. Appropriate technology: antenatal care. Int J Gynaecol Obstet 1998; 63 (Suppl 1):S915.

15.Bergsjo P. What is the evidence for the role of antenatal care strategies in the reduction of maternal mortality and morbidity? In: Brouwere VD, Lerberghe WV, editors. Safe motherhood strategies. A review of the evidence. $1^{\text {st }}$ ed. Belgium: Studies in Health Services Organization and Policy ITG Press; 2001. p. 35-54. 
16. Carroli G, Villar J, Piaggio G, et al. WHO systematic review of randomised controlled trials of routine antenatal care. Lancet 2001; 357:1565-70.

17.Goodburn E, Campbell O. Reducing maternal mortality in the developing world: sector-wide approaches may be the key. BMJ 2001; 322:917-20.

18.World Health Organization. WHO Programme to map best reproductive health practice. WHO Antenatal Care Randomized Trial: manual for the implementation of the new model. Geneva: World Health Organization; 2002.

19.Bergsjo P, Villar J. Scientific basis for the content of routine antenatal care. II. Power to eliminate or alleviate adverse newborn outcomes; some special conditions and examinations. Acta Obstet Gynecol Scand 1997; 76:15-25.

20.Halpern R, Barros FC, Victora CG, Tomasi E. Atenção pré-natal em Pelotas, Rio Grande do Sul, Brasil, 1993. Cad Saúde Publica 1998; 14:487-92.
21.Puccini RF, Pedroso GC, Silva EMK, Araújo NS, Silva NN. Eqüidade na atenção pré-natal e ao parto em área da Região Metropolitana de São Paulo, 1996. Cad Saúde Publica 2003; 19:35-45.

22.Ministério da Saúde. Secretaria de Políticas de Saúde. Pré-natal, parto e puerpério. Assistência humanizada à mulher. Brasília; 2002.

23.Villar J, Ba'aqeel H, Piaggio G, et al. WHO antenatal care randomised trial for the evaluation of a new model of routine antenatal care. Lancet 2001; 357:1551-64.

24.Langer A, Nigenda G, Romero M, et al. Conceptual bases and methodology for the evaluation of women's and providers' perception of the quality of the antenatal care in the WHO Antenatal Care Randomised Controlled Trial. Paediatr Perinat Epidemiol 1998; 12 (Suppl 2):98-115.

Recebido em: 5/2/2004 Aceito com modificações em: 27/7/2004

\title{
XXIII CONGRESSO DE OBSTETRÍCIA E GINECOLOGIA DO NORDESTE
}

\section{0 a 13 de novembro de 2004}

\section{Salvador - BA}

\section{Informações: SOGIBA}

\author{
Tel: (71) 331-9666 \\ Fax: (71) 247-4351 \\ e-mail: st-eventos@terra.com.br
}

